\title{
COMPLEMENTED IDEALS IN THE FOURIER ALGEBRA AND THE RADON NIKODYM PROPERTY
}

\author{
BRIAN FORREST
}

\begin{abstract}
Necessary and sufficient conditions are given for an ideal $I(H)$ of the Fourier algebra to be complemented when $H$ is a closed subgroup of $G$. Using the Radon Nikodym property, an example of a group $G$ with a normal abelian subgroup $H$ for which $I(H)$ is not complemented is presented.
\end{abstract}

\section{INTRODUCTION}

The problem of characterizing complemented ideals in the group algebra $L^{1}(G)$ of a locally compact abelian group $G$ has received considerable attention. D. J. Newman showed that if $\Pi$ is the circle group and $H^{1}=\{f \in$ $L^{1}(\Pi) \mid \hat{f}(n)=0$ for every $\left.n<0\right\}$, then $H^{1}$ is not complemented in $L^{1}(\Pi)$ [20]. Later in [23], Rudin proved that $I$ is complemented in $L^{1}(\Pi)$ if and only if $I=I(A)=\left\{f \in L^{1}(\Pi) \mid \hat{f}(n)=0\right.$ for every $\left.n \in A\right\}$ with $A=\bigcup_{i=1}^{n} a_{i} \mathbb{Z}+b_{i}$.

In his memoir [22], Rosenthal showed that for an arbitrary locally compact abelian group a necessary condition for $I$ to be complemented is that $I=I(A)$, where $A$ is a closed element of the coset ring of $\widehat{G}$. Alspach and Matheson completed the characterization for $G=\mathbb{R}$ by proving that $I$ is complemented if and only if $I=I(A)$, where $A=\bigcup_{i=1}^{n}\left(\tau_{i} \mathbb{Z}+\beta_{i}\right) \backslash F$, the $\tau_{i}$ 's are pairwise rationally dependent and $F$ is finite [2].

Alspach, Matheson, and Rosenblatt examined the problem for arbitrary locally compact abelian groups and were successful in giving a necessary and sufficient condition for an ideal with a discrete hull to be complemented [3]. (Their proof was incorrect but was subsequently corrected in [4].) They also developed an inductive procedure which Alspach exploited to characterize the complemented ideals in $L^{1}\left(\mathbb{R}^{2}\right)[1]$.

By identifying $L^{1}(G)$ with $A(\widehat{G})$, we can view these studies as a series of investigations into the ideal structure of the Fourier algebra of a locally compact abelian group. In this paper we will examine the complementation problem for the Fourier algebra of an arbitrary locally compact group $G$. In particular, we will focus on the question of identifying those closed subgroups $H$ of $G$ which are such that $I(H)$ is complemented.

Received by the editors July 11,1990 .

1980 Mathematics Subject Classification (1985 Revision). Primary 43A07, 43A15; Secondary $46 \mathrm{~J} 10$.

Key words and phrases. Fourier algebra, complemented ideals, coset ring, projection, Radon Nikodym property. 
In $\S 3$, we verify a conjecture of Herz $[12$, p. 161] by presenting an example of a locally compact group $G$ and of a closed subgroup $H$ for which $I(H)$ is not complemented in $A(G)$. Our proof is geometric in nature. It shows that the question of spectral synthesis which Herz believed to be at the heart of the matter is not the true barrier to complementation.

\section{Preliminaries}

Throughout this paper $G$ will denote a locally compact group with a fixed left Haar measure $d x . \Sigma_{G}$ will denote the class of equivalence classes of strongly continuous unitary representations of $G . \widehat{G}$ is the set of irreducible classes in $\Sigma_{G}$.

For each $\pi \in \Sigma_{G}, \xi, \eta \in \mathscr{H}_{\pi}$, the Hilbert space associated with $\pi$, define $u_{\pi, \xi, \eta}(x)=\langle\pi(x) \xi, \eta\rangle$. Let $B(G)$ be the collection of all such coefficient functions. It is well known that $B(G)$ is the linear span of continuous positive definite functions on $G$. Furthermore $B(G)$ is an algebra with respect to pointwise addition and multiplication.

Define a norm $\|\cdot\|_{B(G)}$ on $B(G)$ by

$$
\|u\|_{B(G)}=\inf _{\substack{\pi \in \Sigma, \xi_{i}, \eta_{i} \in \mathscr{H}_{\pi}}}\left\{\sum_{i=1}^{\infty}\left\|\xi_{i}\right\|\left\|\eta_{i}\right\| \mid u(x)=\sum_{i=1}^{\infty}\left\langle\pi(x) \xi_{i}, \eta_{i}\right\rangle\right\} .
$$

With the norm $\|\cdot\|_{B(G)}, B(G)$ becomes a commutative Banach algebra called the Fourier-Stieltjes algebra. $B(G)$ was first introduced and studied by Eymard in [9].

Define $\lambda(x): L^{2}(G) \rightarrow L^{2}(G)$ by $\lambda(x) f(y)=f\left(x^{-1} y\right)$ for each $f \in L^{2}(G)$, $x, y \in G$. Then $\lambda \in \Sigma_{G}$ is called the left regular representation of $G$. Let $A(G)=\left\{u(x) \mid u(x)=\langle\lambda(x) f, g\rangle, f, g \in L^{2}(G)\right\}$. Then $A(G)$ is a closed ideal of $B(G)\left[9\right.$, p. 208]. With respect to $\|\cdot\|_{A(G)}=\|\cdot\|_{\left.B(G)\right|_{A(G)}}, A(G)$ is a commutative Banach algebra with maximal ideal space $\Delta(A(G))=G$.

Let $u \in A(G)$. Define $\left(L_{x} u\right)(y)=u\left(x^{-1} y\right)$. Then $L_{x} u \in A(G)$ and $\left\|L_{x} u\right\|_{A(G)}=\|u\|_{A(G)}$. Let $A, B \subset G$ be closed. Define $\rho(A, B)=\{u \in$ $B(G) \mid u(A)=1, u(B)=0\}$. Let

$$
s(A, B)= \begin{cases}\inf \left\{\|u\|_{B(G)} \mid u \in \rho(A, B)\right\} & \text { if } \rho(A, B) \neq \varnothing \\ \infty & \text { if } \rho(A, B)=\varnothing .\end{cases}
$$

Let $I$ be an ideal of $A(G)$. Let $Z(I)=\{x \in G \mid u(x)=0$ for every $u \in I\}$. Then $Z(I)$ is a closed subset of $G$. Given $A \subset G$ closed, $I(A)=\{u \in$ $A(G) \mid u(x)=0$ for every $x \in A\}$. A closed set $A \subset G$ is called a set of spectral synthesis (or simply an $s$-set) if $Z(I)=A$ implies that $I$ is dense in $I(A)$.

Let $\mathscr{Z}(G)$ denote the center of $G$. If $H$ is a subgroup of $G, C_{G}(H)$ will denote the centralizer of $H$. The following classes of locally compact groups will be considered:

$[Z]-C e n t r a l$ topological groups- $G / Z(G)$ is compact.

[SIN]-Small Invariant Neighborhood groups-every neighborhood of the identity contains a compact neighborhood which is invariant under all inner automorphisms.

[IN]-Invariant Neighborhood groups-there exists a compact neighborhood of the identity which is invariant under all inner automorphisms. 
$[\mathrm{FC}]^{-}$- Topologically Finite Conjugacy Class groups-the closure of each conjugacy class is compact.

For properties of these classes see [21].

\section{THE COMPLEMENTED IDEAL PROPERTY FOR CLOSED SUBGROUPS}

Definition 3.1. A closed ideal $I$ in $A(G)$ is said to be complemented if there exists a continuous projection $P$ of $A(G)$ onto $I$. Let $H$ be a closed subgroup of $G . H$ is said to have the complemented ideal property (CIP)) in $G$ if $I(H)$ is complemented in $A(G)$. Let $\operatorname{CIP}(G)=\{H \mid H$ has CIP in $G\}$.

Proposition 3.2. Let $H$ be an open subgroup of $G$. Then $H \in \operatorname{CIP}(G)$. In particular, if $G$ is a discrete group, then every subgroup of $G$ belongs to $\operatorname{CIP}(G)$. Proof. Since $H$ is open, $1_{H} \in B(H)\left[9\right.$, p. 205] and thus $1_{G \backslash H} \in B(G)$. Therefore $A(G)=1_{H} A(G) \oplus 1_{G \backslash H} A(G)$ and $1_{G \backslash H} A(G)=I(H)$.

It should be noted that if $A \subset G$, then $1_{A} \in B(G)$ if and only if $A$ belongs to $\mathscr{R}(G)$, the ring of subsets of $G$ generated by open cosets of $G$. Hence if $A \in \mathscr{R}(G)$, then as in Proposition 3.2, we see that $I(A)$ is complemented in $A(G)$. For abelian groups, Rosenthal has shown for an ideal $I \subset A(G)$ to be complemented, $Z(I)$ must belong to $\mathscr{R}\left(G_{d}\right)$, the coset ring of $G$ with the discrete topology [22]. Though it is very likely that Rosenthal's result holds for all locally compact groups we are unable to prove this. This does however motivate us to consider the question of identifying those subgroups with the CIP. The next proposition is our key tool in this regard.

Proposition 3.3. Let $H$ be a closed subgroup. Then $H \in \operatorname{CIP}(G)$ if and only if there exists a continuous linear map $\Gamma: A(H) \rightarrow A(G)$ such that $\Gamma u_{\mid H}=u$ for every $u \in A(H)$.

Proof. Assume that $H \in \operatorname{CIP}(G)$. Let $Q$ be a continuous projection of $A(G)$ onto $I(H)$. Let $u \in A(H)$. Let $v \in A(G)$ be such that $v_{\mid H}=u$ and $\|v\|_{A(G)}=$ $\|u\|_{A(H)}[13$, Theorem 1].

Define $\Gamma u=v-Q v$. Then $\|\Gamma u\|_{A(G)} \leq(1+\|Q\|)\|u\|_{A(H)} . \Gamma$ is well defined since $\left(v_{1}-Q v_{1}\right)-(v-Q v)=\left(v_{1}-v\right)-Q\left(v_{1}-v\right)=0$ for any other extension $v_{1}$ of $u$.

Let $u_{1}, u_{2} \in A(H)$, and $\alpha_{1}, \alpha_{2} \in \mathbb{C}$. Choose any two extensions $v_{1}$ and $v_{2}$ of $u_{1}$ and $u_{2}$ respectively. Then

$$
\Gamma\left(\alpha_{1} u_{1}+\alpha_{2} u_{2}\right)=\left(\alpha_{1} v_{1}+\alpha_{2} v_{2}\right)-Q\left(\alpha_{1} v_{1}+\alpha_{2} v_{2}\right)=\alpha_{1} \Gamma\left(u_{1}\right)+\alpha_{2} \Gamma\left(u_{2}\right) .
$$

Hence $\Gamma$ is linear.

Conversely, assume that $\Gamma: A(H) \rightarrow A(G)$ is a continuous linear extension map. For $v \in A(G)$ define $Q(v)=v-\Gamma\left(v_{\mid H}\right)$. Then $\|Q\| \leq 1+\|\Gamma\|, Q(v) \epsilon$ $I(H)$ and since $v \in I(H)$ implies $v_{\mid H}=0, Q(v)=v$ for every $v \in I(H)$.

The following proposition is the starting point of [3]. It is not stated as such, but the essential ideas of the proof are included as a remark [3, p. 257].

Proposition 3.4. Let $G$ be an abelian locally compact group. Let $H$ be a closed subgroup of $G$. Then there exists a linear isometry $\Gamma: A(H) \rightarrow A(G)$ such that $\Gamma u_{\mid H}=u$ for every $u \in A(H)$. In particular, $H \in \operatorname{CIP}(G)$.

Definition 3.5. A representation $\pi \in \Sigma_{G}$ is said to be completely reducible if $\pi=\sum_{\alpha \in \mathscr{U}} \bigoplus \sigma_{\alpha}$ where $\sigma_{\alpha} \in \widehat{G}$. 
It is well known that if $G$ is abelian, then the left regular representation $\lambda_{G}$ is completely reducible if and only if $G$ is compact; cf. [25].

Theorem 3.6. Let $G$ be a locally compact group. Let $H$ be a closed subgroup of $G$ such that the left regular representation of $H$ is completely reducible. Then there exists a linear isometry $\Gamma: A(H) \rightarrow A(G)$ such that $\Gamma u_{\left.\right|_{H}}=u$ for every $u \in A(H)$.

Proof. Since $A(G)_{\left.\right|_{H}}=A(H), \lambda_{\left.G\right|_{H}}$ is quasiequivalent to $\lambda_{H}$ [5, p. 27]. Therefore there exists cardinal numbers $\eta_{G}$ and $\eta_{H}$ such that $\eta_{G}\left(\lambda_{\left.G\right|_{H}}\right)$ is unitarily equivalent to $\eta_{H} \lambda_{H}$. Let $T: \eta_{G} \mathscr{H}_{\lambda_{G}} \rightarrow \eta_{H} \mathscr{H}_{\lambda_{H}}$ be an intertwining operator.

As $\lambda_{H}$ is completely reducible, we can write

$$
\lambda_{H}=\sum_{\alpha \in \mathscr{I}} \bigoplus m_{\alpha} \sigma_{\alpha} \quad \text { and } \quad L^{2}(H)=\mathscr{H}_{\lambda_{H}}=\sum_{\alpha \in \mathscr{I}} \bigoplus m_{\alpha} \mathscr{H}_{\sigma_{\alpha}},
$$

where $\sigma_{\alpha} \in \widehat{H}$. It follows that $T^{-1} \mathscr{H}_{\sigma_{\alpha}}$ is a closed subspace of $\eta_{G} \mathscr{H}_{\lambda_{G}}$.

Let $A_{\sigma_{\alpha}}$ denote the closure in $B(H)$ of the space $F_{\sigma_{\alpha}}$, the linear span of the coefficient functions of $\sigma_{\alpha}$. Since $\sigma_{\alpha}$ is irreducible, $A_{\sigma_{\alpha}}$ is linearly isomorphic isometric to $\mathscr{H}_{\sigma_{\alpha}} \hat{\otimes} \mathscr{H}_{\sigma_{\alpha}}$ [5, 2éme partie] where $\hat{\otimes}$ denotes the projective tensor product. For $\xi, \eta \in \mathscr{H}_{\sigma_{\alpha}}$ define

$$
\Gamma_{\sigma_{\alpha}}\left(\left\langle\sigma_{\alpha}(\cdot) \xi, \eta\right\rangle\right)=\left\langle\eta_{G} \lambda_{G}(\cdot) T^{-i} \xi, T^{-1} \eta\right\rangle .
$$

Then $\Gamma_{\sigma_{\alpha}}$ extends to a linear map of $F_{\sigma_{\alpha}}=\mathscr{H}_{\sigma_{\alpha}} \otimes \mathscr{H}_{\sigma_{\alpha}}$ into $A_{\eta_{G} \lambda_{G}}$ with

$$
\begin{aligned}
\left\|\Gamma_{\sigma_{\alpha}}\left(\sum_{i=1}^{n}\left\langle\sigma_{\alpha}(\cdot) \xi_{i}, \eta_{i}\right\rangle\right)\right\|_{B(G)} & \leq \sum_{i=1}^{n}\left\|T^{-1} \xi_{i}\right\|_{\mathscr{P}_{\eta_{G}{ }^{\lambda} G}}\left\|T^{-1} \eta_{i}\right\|_{\mathscr{H}_{\eta_{G}{ }^{\lambda} G}} \\
& =\sum_{i=1}^{n}\left\|\xi_{i}\right\|_{\mathscr{H}_{\sigma_{\alpha}}}\left\|\eta_{i}\right\|_{\mathscr{H}_{\sigma_{\alpha}}} .
\end{aligned}
$$

Hence

$$
\left\|\Gamma_{\sigma_{\alpha}}\left(\sum_{i=1}^{n}\left\langle\sigma_{\alpha}(\cdot) \xi_{i}, \eta_{i}\right\rangle\right)\right\|_{B(G)} \leq\left\|\sum_{i=1}^{n}\left\langle\sigma_{\alpha}(\cdot) \xi_{i}, \eta_{i}\right\rangle\right\|_{A(H)} .
$$

Therefore, $\Gamma_{\sigma_{\alpha}}$ extends to a norm nonincreasing linear map of $A_{\sigma_{\alpha}}$ into $A_{\eta_{G} \lambda_{G}}$.

Since $T_{\eta_{G} \lambda_{G} \mid H}=\eta_{H} \lambda_{H} T$,

$$
\left\langle\sigma_{\alpha}(h) \xi, \eta\right\rangle=\left\langle\eta_{G} \lambda_{G}(h) T^{-1} \xi, T^{-1} \eta\right\rangle=\Gamma_{\sigma_{\alpha}}\left(\left\langle\sigma_{\alpha}(\cdot) \xi, \eta\right\rangle\right)(h)
$$

for every $\xi, \eta \in \mathscr{H}_{\sigma_{\alpha}}$. As convergence in $B(H)$ and $B(G)$ implies uniform convergence [9, p. 182], $\Gamma_{\sigma_{\alpha}} u_{\left.\right|_{H}}=u$ for every $u \in A_{\sigma_{\alpha}}$. Furthermore, $\Gamma_{\sigma_{\alpha}}$ is an isometry.

Finally $A(H)=l_{1}-\bigoplus_{\alpha \in \mathcal{F}} A_{\sigma_{\alpha}}[5$, p. 39]. Define $\Gamma: A(H) \rightarrow B(G)$ by

$$
\Gamma u=\sum_{\alpha \in \mathcal{I}} \Gamma_{\sigma_{\alpha}} u_{\sigma_{\alpha}}
$$

where $u=\sum_{\alpha \in \mathcal{I}} u_{\sigma_{\alpha}}$ is the unique decomposition of $u$.

Note that

$$
\begin{aligned}
\|\Gamma u\|_{B(G)} & =\left\|\sum_{\alpha \in \mathcal{I}} \Gamma_{\sigma_{\alpha}} u_{\sigma_{\alpha}}\right\|_{B(G)}=\sum_{\alpha \in \mathscr{I}}\left\|u_{\sigma_{\alpha}}\right\|_{B(H)} \\
& =\sum_{\alpha \in \mathscr{I}}\left\|u_{\sigma_{\alpha}}\right\|_{A(H)}=\|u\|_{A(H)} .
\end{aligned}
$$


Also since $\Gamma_{\sigma_{\alpha}} u_{\left.\sigma_{\alpha}\right|_{H}}=u_{\left.\sigma_{\alpha}\right|_{H}}$ for every $\alpha \in \mathscr{I}, \Gamma u_{\left.\right|_{H}}=u$ for every $u \in A(H)$. Therefore $\Gamma$ is a linear isometry of $A(H)$ into $B(G)$. But $\Gamma u \in A_{\eta_{G} \lambda_{G}}=A(G)$ $[5$, p. 27].

Corollary 3.7. Let $H$ be a compact subgroup of $G$. Then there exists a linear isometry $\Gamma: A(H) \rightarrow A(G)$ such that $\Gamma u_{\left.\right|_{H}}=u$ for every $u \in A(H)$. Furthermore, $H \in \operatorname{CIP}(G)$.

Proof. It is well known that if $H$ is compact, then $\lambda_{H}$ is completely reducible.

Corollary 3.7 is due to Herz [12, Theorem 4]. His proof involves techniques from the theory of induced representations, an approach which we shall exploit later. However, since there are many noncompact groups for which $\lambda_{G}$ is completely reducible, Theorem 3.6 is a proper extension of Herz's result. Moreover, the geometric nature of the above proof sheds new light on the complementation problem. We will develop this further.

Proposition 3.8. Let $G$ be a locally compact group. Let $H$ be a closed subgroup of $G$ and let $\pi$ be a completely reducible representation of $H$. If every $u \in A_{\pi}$ extends to some $v \in B(G)$, then there exists a linear isometry $\Gamma: A_{\pi} \rightarrow B(G)$ for which $\Gamma u_{\left.\right|_{H}}=u$.

Proof. Let $\omega$ denote the universal representation of $G$. Then $A_{\omega_{\left.\right|_{H}}}$ contains any element of $B(H)$ which extends to $B(G)$. By assumption $A_{\pi} \subseteq A_{\omega_{\left.\right|_{H}}}$. Hence $\pi$ is quasiequivalent to a subrepresentation of $\omega_{\left.\right|_{H}}[5$, p. 40]. The remainder of the proof is similar to the proof of Theorem 3.6.

Example 3.9. (i) Let $G$ be a unimodular group. Let

$$
\widehat{G}_{d}=\{\pi \in \widehat{G} \mid \pi \text { is square integrable }\} .
$$

Let $A_{d}(G)$ denote the closed subspace of $A(G)$ generated by the extreme points of the unit ball of $A(G)$, Mauceri [19] has shown that

$$
A_{d}(G)=l_{1}-\bigoplus_{\pi \in \widehat{G}_{d}} A_{\pi}
$$

and that there exists a closed subspace $A_{c}(G)$ such that $A(G)=A_{d}(G) \oplus A_{c}(G)$.

Let $H$ be a closed subgroup of $G$. Then Proposition 3.8 establishes a linear isometry $\Gamma: A_{d}(H) \rightarrow A(G)$ for which $\Gamma_{u_{\left.\right|_{H}}}=u$ for every $u \in A_{d}(H)$. Therefore if $H \notin \operatorname{CIP}(G)$, the difficulty lies within $A_{c}(H)$.

(ii) Let $\operatorname{AP}(G)$ denote the space of continuous almost periodic functions on $G$. Let $G^{\text {ap }}$ denote the almost periodic compactification of $G$. If $\varphi: G \rightarrow G^{\text {ap }}$ is the canonical homomorphism, then $\operatorname{ker} \varphi$ is such that every $f \in \operatorname{AP}(G)$ is constant on cosets of $\operatorname{ker} \varphi$.

Assume that $H$ is a compact subgroup of $G$. Then $\varphi(H) \cong H /(\operatorname{ker} \varphi \cap H)$ is a compact subgroup of $G^{\text {ap }}$. Moreover $A(\varphi(H))$ is isometrically isomorphic to the closed subalgebra of $A(H)$ which consists of functions which are constant on cosets of $\operatorname{ker} \varphi \cap H$. We will denote this algebra by $A_{\varphi}(H)$.

It follows from Theorem 3.6 that there exists a linear isometry $\Gamma_{1}: A(\varphi(H))$ $\rightarrow A\left(G^{\mathrm{ap}}\right)$ for which $\Gamma_{1}: u_{\left.\right|_{\varphi(H)}}=u$. This provides us with the means of constructing a linear isometry $\Gamma: A_{\varphi}(H) \rightarrow \operatorname{AP}(G) \cap B(G)$ for which $\Gamma_{u_{\left.\right|_{H}}}=u$. 
This follows since Eymard proved that $\operatorname{AP}(G) \cap B(G)$ can be identified in a natural way with $A\left(G^{\text {ap }}\right)[9$, p. 203].

In case $G$ is noncompact, $(\operatorname{AP}(G) \cap B(G)) \cap A(G)=\{0\}$. Hence the extending map is entirely distinct from the map constructed in the proof of Theorem 3.6. Finally, we note that if $G$ is abelian, then $\operatorname{ker} \varphi \cap H=\{e\}$. Hence all of $A(H)$ extends to $\operatorname{AP}(G) \cap B(G)$.

(iii) Let $H$ be the " $a x+b$ " group. We recall that $H$ can be realized as the matrix group

$$
\left\{\left(\begin{array}{ll}
a & b \\
0 & 1
\end{array}\right) \mid a \in \mathbb{R}^{+}, b \in \mathbb{R}\right\} .
$$

Then $\lambda_{H}$ is completely reducible, cf. [16]. For any $n \geq 2, G=\mathrm{GL}(n, \mathbb{R})$ contains subgroups isomorphic to $H$. Let $H_{1}$ be such a group. Then there is a linear isometry $\Gamma: A\left(H_{1}\right) \rightarrow A(G)$ for which $\Gamma_{u_{\left.\right|_{H_{1}}}}=u$. Consequently, $H_{1} \in \operatorname{CIP}(G)$.

Definition 3.10. A Banach space $X$ is said to possess the Radon-Nikodym property if for any finite measure space $(\Omega, \Sigma, \mu)$ and any $\mu$-continuous vector measure $L: \Sigma \rightarrow X$ of bounded total variation, there exists a Bochner integrable function $g: \Sigma \rightarrow X$ such that

$$
L(E)=\int_{E} g d \mu \quad \text { for every } E \in \Sigma \quad \text { (cf. [8]). }
$$

In [7, p. 535] Chu showed that the pre-dual of a von Neumann algebra has the RNP if and only if every bounded norm closed convex subset is the closed convex hull of its extreme points.

Granirer and Leinert proved that if $K \subset G$ is compact, $A_{K}(G)=\{u \in$ $A(G) \mid \operatorname{supp} u \subseteq K\}$ has the RNP [11, p. 464]. K. Taylor showed that $A(G)$ has the RNP if and only if $\lambda_{G}$ is completely reducible [25]. As it is easy to see that the RNP is preserved by linear isometries the previous example shows that if $n \geq 2$, then $G=\mathrm{GL}(n, \mathbb{R})$ has closed subspaces with the RNP which are not of the form $A_{K}(G)$ for some compact set $K$ even though $A(G)$ does not have the RNP, cf. [25]. However, we have:

Proposition 3.11. Let $G$ be a locally compact group. Let $H$ be a closed subgroup of $G$ such that $\lambda_{H}$ is completely reducible. Then $I(H)$ has the RNP if and only if $A(G)$ has the RNP.

Proof. If $A(G)$ has the RNP, then so does every closed subspace.

Conversely, assume that $I(H)$ has the RNP. Since $\lambda_{H}$ is completely reducible, $A(H)$ has the RNP. It follows from [10, Lemma 3.9] that $A(G) / I(H)$ has the RNP. Hence $A(G)$ has the RNP; cf. [8, p. 211].

Proposition 3.12. Let $G$ be a locally compact group for which $A(G)$ has the RNP. Let $H$ be a closed subgroup of $G$. if $H \in \operatorname{CIP}(G)$, then $A(H)$ has the RNP.

Proof. Let $P$ be a projection of $A(G)$ onto $I(H)$. Then $(1-P)(A(G))$ has the RNP. Define $\Gamma_{0}:(1-P)(A(G)) \rightarrow A(H)$ by $\Gamma_{0} u=u_{\left.\right|_{H}}$ for every $u \in$ $(1-P)(A(G)) . \quad \Gamma_{0}$ is linear and $\left\|\Gamma_{0}\right\| \leq 1$. If we let $\Gamma: A(H) \rightarrow A(G)$ be the map constructed as in the proof of Proposition 3.3, then $\Gamma=\Gamma_{0}^{-1}$ and $\|\Gamma\|<\infty$. Therefore, $A(H)$ and $(1-P)(A(G))$ are linearly isomorphic and 
homeomorphic. It follows that every norm closed convex subset of $A(H)$ is the closed convex hull of its extreme points. Therefore, by Chu's result [7], $A(H)$ has the RNP.

Example 3.13. Let $G=\mathbb{R} \odot \mathbb{R}^{+}$be the " $a x+b$ " group. Then $A(G)$ has the RNP. Also $G$ has a closed normal subgroup $H$ which is isomorphic to $\mathbb{R}$. But $A(\mathbb{R})$ does not have the RNP [cf. 25]. Hence by Proposition 3.12, $I(H)$ is not complemented in $A(G)$.

Observe that $\mathbb{R}$ is an $s$-set in $G$ [24] hence the problem of spectral synthesis hinted at in [12, p. 161] is avoided. In addition, the above example shows that the assumptions that a subgroup is normal or abelian or that the group $G$ is amenable will not insure complementation. This seems to indicate that the complementation problem is much more difficult in the noncommutative setting than in the abelian case which is in its own right far from trivial.

\section{PRODUCT GROUPS AND CENTRAL SUBGROUPS}

Theorem 4.1. Let $H$ be a closed subgroup of a separable locally compact $\sigma$ compact group $G$. Let $C$ be a subgroup of $C_{G}(H)$ and $K=C H$. If $K$ is closed in $G$, then there exists a linear isometry $\Gamma: A(H) \rightarrow A(K)$ such that $\Gamma u_{\mid H}=u$ for every $u \in A(H)$. In particular, $H \in \operatorname{CIP}(K)$.

Proof. Observe that $K / H \cong C / C \cap H$. Let $\varphi: K / H \rightarrow C / C \cap H$ be the canonical isomorphism. Let $\psi_{1}: C / C \cap H \rightarrow C$ be a Borel cross section [cf. 18, p. 102]. Define $\psi: K / H \rightarrow K$ by $\psi=\psi_{1} \circ \xi$. Then $\psi$ is a Borel cross section and $\psi(\xi) \in C$ for every $\xi \in K / H$.

Let $\Omega$ be a compact subset of $K / H$ with $\mu_{K / H}(\Omega)>0$. Let $x \in K$ and $\xi \in K / H$. Define $\xi^{x}=\pi\left(x^{-1} y\right)$, where $\pi: K \rightarrow K / H$ is the canonical homomorphism and $y \in K$ is such that $\pi(y)=\xi$.

Define $\tau: K \times K / H \rightarrow H$ by

$$
\tau(x, \xi)=\psi(\xi)^{-1} x \psi\left(\xi^{x}\right) .
$$

For each $u \in A(H) \cap C_{00}(H)$, let

$$
\Gamma_{0} u(x)=\frac{1}{\mu_{K / H}(\Omega)} \int_{K / H} 1_{\Omega}\left(\xi^{x}\right) 1_{\Omega}(\xi) \mu(\tau(x, \xi)) d \mu_{K / H}(\xi) .
$$

Then $\operatorname{supp}\left(\Gamma_{0} u\right)$ is compact. Furthermore $\Gamma_{0} u \in A(K)$ with $\left\|\Gamma_{0} u\right\|_{A(K)} \leq$ $\|u\|_{A(H)} ;$ cf. [13, p. 114].

Let $h \in H$. Then $\xi^{h}=\xi$ and since $\psi(\xi) \in C$,

$$
\tau(h, \xi)=\psi(\xi)^{-1} h \psi(\xi)=h .
$$

Therefore $\Gamma_{0} u(h)=u(h)$. Hence $\Gamma_{0} u_{\left.\right|_{H}}=u$ and $\left\|\Gamma_{0} u\right\|_{A(K)}=\|u\|_{A(H)}$. As $A(H) \cap C_{00}(H)$ is dense in $A(H), \Gamma_{0}$ can be extended to an isometry $\Gamma: A(H) \rightarrow A(K)$.

Corollary 4.2. Let $H$ be a closed central subgroup of a locally compact group $G$. Then there exists an isometry $\Gamma: A(H) \rightarrow A(G)$ for which $\Gamma u_{\left.\right|_{H}}=u$ for every $u \in A(H)$.

Proof. (i) Assume that $G$ is separable and $\sigma$-compact. Then the result follows immediately from the proof of Theorem 4.2. 
(ii) Suppose that $G$ is $\sigma$-compact. Let $K$ be a compact normal subgroup of $G$ such that $G / K$ is separable. Let $\varphi: G \rightarrow G / K$ be the canonical homomorphism. By (i), there exists a linear isometry $\Gamma_{0_{K}}: A(\varphi(H)) \rightarrow A(G / K)$ such that $\Gamma_{0_{K}} u_{\left.\right|_{\varphi(H)}}=u$. But $\varphi(H) \cong H /(K \cap H)$. Hence $A(\varphi(H))$ can be naturally identified with the subalgebra of $A(H)$ consisting of functions which are constant on cosets of $(K \cap H)$. Similarly $A(G / K)$ can be identified with the subalgebra of $A(G)$ consisting of functions which are constant on cosets of $K$. Composition of these mappings gives us a linear isometry $\Gamma_{K}$ from $A(H /(H \cap K)) \subseteq A(H)$ into $A(G / K) \subseteq A(G)$ for which $\Gamma_{K} u_{\left.\right|_{H}}=u$.

Assume that $K_{1}$ is any other compact normal subgroup of $G$ such that $G / K_{1}$ is separable and that $K_{1} \subseteq K$. Observe that $A(H / K \cap H) \subseteq A\left(H / K_{1} \cap H\right)$. Moreover, because the process used to produce the isometry $\Gamma$ in Theorem 4.1 is essentially an averaging procedure, a careful examination will show that $\Gamma_{K_{1}}$ is an extension of $\Gamma_{K}$. Since every $u \in A(H)$ belongs to $A\left(H / K_{1} \cap H\right)$ for some such compact normal group $K_{1}$, the desired isometry is obtained by an obvious directed limit of the $\Gamma_{K}$ 's.

(iii) Assume that $G$ is any locally compact group and that $H$ is any closed subgroup which is also $\sigma$-compact. Then there exists an open $\sigma$-compact group $G_{0} \subset G$ such that $H \subseteq G_{0}$. Identify $A\left(G_{0}\right)$ with the obvious subalgebra of $A(G)$ and apply (ii) to obtain the desired isometry.

Corollary 4.2 is due to Herz [12, Theorem 5]. His proof is for the case $G$ separable, metric and $\sigma$-compact (see [12, p. 161]). For the sake of completeness we have outlined how the separability assumption can be dropped, something which Herz asserts but does not exhibit. In fact, a careful modification of the above procedure will allow us to remove the separability assumption from the statement of Theorem 4.1 which for the rest of the paper we shall assume has been done.

Since we do not know of any way to extend Corollary 4.2 to non- $\sigma$-compact groups, we are not able to obtain Proposition 3.4 as a corollary to the above result.

Let $G_{1}$ and $G_{2}$ be locally compact groups. Let $u_{i} \in A\left(G_{i}\right)$. Define $u \in$ $A\left(G_{1} \times G_{2}\right)$ by $u(x, y)=u_{1}(x) u_{2}(y)$. In this manner we get a linear map $\psi: A\left(G_{1}\right) \hat{\otimes} A\left(G_{2}\right) \rightarrow A\left(G_{1} \times G_{2}\right)$. Furthermore $\psi$ has a dense range and $\|\psi\| \leq$ $1 ;$ cf. [17].

Losert proved that if the irreducible representations of either $G_{1}$ or $G_{2}$ are bounded, then $\psi$ is surjective and $\left\|\psi^{-1}\right\|=\min \left(d_{1}, d_{2}\right)$ where $d_{i}=$ $\sup _{\pi \in \widehat{G}_{i}} \operatorname{dim} \pi$. Consequently if either $G_{1}$ or $G_{2}$ is abelian, then $\psi$ is an isometry.

Conversely, if $\psi$ is a surjection, then one of the $G_{i}$ 's must have an open abelian subgroup of finite index and $\psi$ is an isometry only if one of the $G_{i}$ 's is abelian.

Proposition 4.3. Let $G=G_{1} \times G_{2}$. Then for each $i=1,2$, there exists a linear isometry $\Gamma_{i}: A\left(G_{i}\right) \rightarrow A(G)$ such that $\Gamma_{i} u_{\left.\right|_{G_{i}}}=u$ for each $u \in A\left(G_{i}\right)$. Consequently $G_{i} \in \operatorname{CIP}(G)$.

Proof. Let $u_{2} \in A\left(G_{2}\right)$ be such that $u_{2}\left(e_{2}\right)=1$ and $\left\|u_{2}\right\|_{A\left(G_{2}\right)}=1$. Then $\Gamma_{1} v=\psi\left(v \otimes u_{2}\right)$ for every $v \in A\left(G_{1}\right)$ determines the desired map. $\Gamma_{2}$ is obtained in a similar fashion. 
Lemma 4.4. Let $G=G_{1} \times G_{2}$. Let $A=A_{1} \times A_{2}$ where $A_{i}$ is closed in $G_{i}$. Assume that $A$ is a set of spectral synthesis in $G$. Then $I_{G}(A)$ is the closed linear span $\langle J\rangle^{-}$of $J=\left\{\psi\left(I_{G_{1}}\left(A_{1}\right) \otimes\left(G_{2}\right)\right) \cup \psi\left(A\left(G_{1}\right) \otimes I_{G_{2}}\left(A_{2}\right)\right)\right\}$.

Proof. Clearly $J \subset I_{G}(A)$. Therefore, $\langle J\rangle^{-} \subseteq I_{G}(A)$ as well. Let $v \in A(G)$. Then there exists a net

$$
\left\{\sum_{i=1}^{n_{\alpha}} w_{\alpha_{i}} \otimes z_{\alpha_{i}}\right\}_{\alpha \in \mathscr{U}}
$$

in $A\left(G_{1}\right) \otimes A\left(G_{2}\right)$ such that

$$
v=\lim _{\alpha} \psi\left(\sum_{i=1}^{n_{\alpha}} w_{\alpha_{i}} \otimes z_{\alpha_{i}}\right) .
$$

Assume that $u_{1} \in I_{G_{1}}\left(A_{1}\right)$ and $u_{2} \in A\left(G_{2}\right)$. Then

$$
\psi\left(u_{1} \otimes u_{2}\right) v=\lim _{\alpha} \psi\left[\sum_{i=1}^{n_{\alpha}}\left(u_{1} w_{\alpha_{i}}\right) \otimes\left(u_{2} z_{\alpha_{i}}\right)\right] .
$$

Hence $\psi\left(u_{1} \otimes u_{2}\right) v \in\langle J\rangle^{-}$. Similarly if $u_{1} \in A\left(G_{1}\right), u_{2} \in I_{G_{2}}\left(A_{2}\right)$, then $\psi\left(u_{1} \otimes u_{2}\right) v \in\langle J\rangle^{-}$. Therefore $\langle J\rangle^{-}$is a closed ideal in $A(G)$.

Assume that $\left(x_{1}, x_{2}\right) \notin A_{1} \times A_{2}$. We may assume that $x_{1} \neq A$. We can find $u_{1} \in I_{G_{1}}\left(A_{1}\right)$ such that $u_{1}\left(x_{1}\right)=1$ and $u_{2} \in A\left(G_{2}\right)$ with $u_{2}\left(x_{2}\right)=1$. Since $\psi\left(u_{1} \otimes u_{2}\right)\left(x_{1}, x_{2}\right)=1,2\left(\langle J\rangle^{-}\right)=A_{1} \times A_{2}=A$ But $A$ is an $s$-set, so $\langle J\rangle^{-}=I_{G}(A)$.

Proposition 4.5. Let $G=G_{1} \times G_{2}$, where $G_{1}$ has an abelian subgroup of finite index. Let $A=A_{1} \times A_{2}$, where $A_{i}$ is closed in $G_{i}$. Assume that $A$ is set of spectral synthesis in $G$. Suppose the $I_{G_{i}}\left(A_{i}\right)$ is complemented in $A\left(G_{i}\right)$ for $i=1,2$. Then $I_{G}(A)$ is complemented in $G$.

Proof. Let $P_{i}$ be a continuous projection of $A\left(G_{i}\right)$ onto $I_{G_{i}}\left(A_{i}\right)$. Define $P: A(G) \rightarrow A(G)$ by

$$
P u=u-\psi\left[\left(\left(1-P_{1}\right) \otimes\left(1-P_{2}\right)\right)\left(\psi^{-1} u\right)\right] .
$$

Note that since $G_{1}$ has an abelian subgroup of finite index $\psi^{-1}$ exists and $\left\|\psi^{-1}\right\|<\infty$. Therefore $P$ is continuous.

Let $u_{i} \in A\left(G_{i}\right)$ and let $\left(x_{1}, x_{2}\right) \in A_{1} \times A_{2}$. If $u=\psi\left(u_{1} \otimes u_{2}\right)$, then

$$
\begin{aligned}
P(u)\left(x_{1}, x_{2}\right) & =u_{1}\left(x_{1}\right) u_{2}\left(x_{2}\right)-\left[\left(1-P_{2}\right) u_{1}\right]\left(x_{1}\right)\left[\left(1-P_{2}\right) u_{2}\right]\left(x_{2}\right) \\
& =u_{1}\left(x_{1}\right) u_{2}\left(x_{2}\right)-u_{1}\left(x_{1}\right) u_{2}\left(x_{2}\right)=0 .
\end{aligned}
$$

Therefore $P u \in I_{G}(A)$ for every $u \in A(G)$.

Let $u_{1} \in I_{G_{1}}\left(A_{1}\right)$ and $u_{2} \in A\left(G_{2}\right)$. Let $u=\psi\left(u_{1} \otimes u_{2}\right)$. Then

$$
\begin{aligned}
\psi\left[\left(1-P_{1}\right) \otimes\left(1-P_{2}\right)\left(\psi^{-1} u\right)\right] & =\psi\left[\left(1-P_{1}\right) u_{1} \otimes\left(1-P_{2}\right) u_{2}\right] \\
& =\psi\left(0 \otimes\left(1-P_{2}\right) u_{2}\right)=0 .
\end{aligned}
$$

Therefore $P u=u$. Similarly if $u_{1} \in A\left(G_{1}\right)$ and $u_{2} \in I_{G_{2}}\left(A_{i}\right)$, then for $u=$ $\psi\left(u_{1} \otimes u_{2}\right), P u=u$. By Lemma 4.4, we have $P u=u$ for every $u \in I_{G}(A)$.

Corollary 4.6. Let $G_{i}$ be a locally compact abelian group for each $i=1,2$. Let $A_{i} \subset G_{i}$ be such that $I_{G_{i}}\left(A_{i}\right)$ is complemented in $A\left(G_{i}\right)$. Then $A=A_{1} \times A_{2}$ is such that $A_{G}(A)$ is complemented in $A\left(G_{1} \times G_{2}\right)$. 
Proof. Since $I_{G_{i}}\left(A_{1}\right)$ is complemented, $A_{i} \in \mathscr{R}\left(G_{i_{d}}\right)$. Therefore $A=A_{1} \times A_{2} \in$ $\mathscr{R}\left(\left(G_{1} \times G_{2}\right)_{d}\right)$. It follows that $A$ is an $s$-set [22]. The corollary is therefore implied by Proposition 4.5.

Lemma 4.7. Let $A, B$ be closed subsets of the locally compact group $G$. Suppose that there exists continuous projections $P$ of $A(G)$ onto $I(A)$ and $Q$ of $A(G)$ onto $I(B)$. If $s(A / B, B / A)<\infty$, then there exists a continuous projection $\Gamma$ of $A(G)$ onto $I(A \cup B)$ with $\|\Gamma\| \leq(s(A \backslash B, B \backslash A)+2)(\|P\|+\|Q\|)$.

Proof. Let $u \in \rho(A \backslash B, B \backslash A)$ with $\|u\|_{B(G)} \leq s(A \backslash B, B \backslash A)+1$. Let $v \in$ $A(G)$. Define $\Gamma v=u P v+(1-u) Q v$. It is easy to see that $\Gamma$ is the desired projection.

Corollary 4.8. Let $A \subset G$ be closed. Let $F$ be finite. If there exists a continuous projection of $A(G)$ onto $I(A)$, then there exists a continuous projection of $A(G)$ onto $I(A \cup F)$.

Proposition 4.9. Let $A, B$ be disjoint closed subsets of $G$. Assume that $s(A, B)$ $<\infty$. Then, there exists a continuous projection of $A(G)$ onto $I(A \cup B)$ if and only if there exists continuous projections of $A(G)$ onto $I(A)$ and $I(B)$ respectively.

Proof. If both $I(A)$ and $I(B)$ are complemented, then by Lemma 4.7, $I(A \cup B)$ is complemented in $A(G)$.

Conversely, assume that $P$ is a continuous projection of $A(G)$ onto $I(A \cup B)$. Let $u \in \rho(A, B)$. Define $Q v=P(u v)+v-u v, Q_{1} v=P((1-u) v)-u v$. Then $Q, Q_{1}$ are the desired projections onto $I(A)$ and $I(B)$ respectively.

Proposition 4.10. Let $G$ be a locally compact group and let $H$ be an open abelian subgroup of $G$ which is of finite index in $G$. If $A \subset G$ is such that $I(A)$ is complemented in $A(G)$, then $A \in \mathscr{R}\left(G_{d}\right)$.

Proof. It follows from Proposition 4.9 that $I(H \cap A)$ is complemented in $A(G)$ by means of a continuous projection $P$. If we identify $A(H)$ with the closed subalgebra of $A(G)$ consisting of functions in $A(G)$ which are zero on $G \backslash H$, the restriction of $P$ to $A(H)$ determines a continuous projection of $A(H)$ onto $I_{H}(H \cap A)$. By Rosenthal's result $H \cap A \in \mathscr{R}\left(H_{d}\right) \subseteq \mathscr{R}\left(G_{d}\right)$.

But $A=\bigcup_{i=1}^{n}\left(x_{i} H \cap A\right)$ for some finite set $x_{1}, \ldots, x_{n} \in G$. By translating and repeating the above argument we get that $x_{i} H \cap A \in \mathscr{R}\left(G_{d}\right)$ and hence that $A \in \mathscr{R}\left(G_{d}\right)$.

Proposition 4.10 is a modest improvement of Rosenthal's result. Modifications of the above argument will yield the following two results.

Proposition 4.11. Let $G$ be a locally compact group with an open abelian subgroup. Let $A \subset G$ be compact. If $I(A)$ is complemented in $A(G)$, then $A \in \mathscr{R}\left(G_{d}\right)$.

Theorem 4.12. Let $G$ be a locally compact group with an open subgroup $H$ which is such that every closed subgroup $H_{1}$ of $H$ belongs to $\operatorname{CIP}(H)$. Let $\mathrm{H}_{2}$ be any closed subgroup of $G$. There exists a linear map $\Gamma: A\left(H_{2}\right) \rightarrow A(G)$ such that $\Gamma u_{\left.\right|_{H_{2}}}=u$ for every $u \in A(G)$ and if $K \subset H_{2}$ is compact, then $\left\|\Gamma_{\left.\right|_{A_{K}}\left(H_{2}\right)}\right\| \leq C_{K}$ for some constant which depends only upon $K$. 
Proof. Let $H_{2}=\bigcup_{\alpha \in \mathscr{A}} x_{2}\left(H \cap H_{2}\right)$. If $u \in A\left(H_{2}\right)$, then we can write $u$ as the formal sum $u=\sum_{\alpha} u_{\alpha}$ where $u_{\alpha}$ is supported on $x_{\alpha}\left(H \cap H_{2}\right)$. Since $H$ is open, if $\mathrm{K} \subset \mathrm{H}_{2}$ is compact, then each $u \in A\left(H_{2}\right)$ supported on $K$ can be written as a finite sum $\sum_{i=1}^{n_{K}} u_{\alpha_{i}}$. Furthermore $n_{K}$ is determined by $K$ independent of $u$ and $\left\|u_{\alpha_{i}}\right\|_{A\left(H_{2}\right)} \leq\|u\|_{A(H)}$.

Clearly $\left(H \cap H_{2}\right) \in \operatorname{CIP}(G)$. Let $\Gamma_{0}$ be the extending map associated with $\left(H \cap H_{2}\right)$ and $C_{1}=\|\Gamma\|$. By repeated translation, we can extend each $u_{\alpha:}$ via $\Gamma_{0}$ to an element $v_{\alpha_{i}} \in A(G)$ with $\left\|v_{\alpha_{i}}\right\|_{A(G)} \leq C\left\|u_{\alpha_{i}}\right\|_{A(H)}$. Let $\Gamma u=\sum_{i=1}^{n_{K}} \Gamma u_{\alpha_{i}}$. Note that $\|\Gamma u\| \leq n_{K} C_{1}\|u\|_{A\left(H_{2}\right)}$. Thus $C_{K}=n_{K} C_{1}$.

This process is easily seen to determine a well-defined extending map from the space $A\left(H_{2}\right) \cap C_{00}\left(H_{2}\right)$ into $A(G)$.

To determine $\Gamma$ on all of $A\left(H_{2}\right)$, extend a basis of $A\left(H_{2}\right) \cap C_{00}\left(H_{2}\right)$ to a basis of $A\left(H_{2}\right)$. For each of the new basis elements simply choose any extension in $A(G)$. Then extend the mapping linearly.

Theorem 4.13. Let $G$ be a locally compact group with an open central subgroup. Let $H$ be a closed subgroup of $G$. Then there exists a linear isometry $\Gamma: A(H) \rightarrow A(G)$ with $\Gamma u_{\left.\right|_{H}}=u$ for every $u \in A(H)$. In particular, $H \in \operatorname{CIP}(G)$.

Proof. Since $Z(G)$ is open, $K=Z(G) H$ is open in $G$ and hence is also closed. We may now apply Theorem 4.1, and the remark following Corollary 4.2.

We are in a position to expand our investigation to a number of large classes of locally compact groups.

Proposition 4.14. Let $G \in[\mathrm{SIN}]$ be almost connected. Let $H$ be a closed subgroup of $G$. If there exists a closed subgroup $C$ with $Z(G) \subseteq C \subseteq C_{G}(H)$ for which $K=H C$ is closed in $G$, then $H \in \operatorname{CIP}(G)$.

Proof. Since $G$ is almost connected, there exists a compact subgroup $K_{1}$ in $G$ and a vector subgroup $V$ such that $V \times K_{1}$ is of finite index in $G$ [21, p. 698]. If $K$ is closed in $G$, then $K \cap\left(V \times K_{1}\right)$ is closed in $V \times K$. Furthermore $K \cap\left(V \times K_{1}\right)$ is of finite index in $K$ and there exists a compact subgroup $K^{*}$ of $K_{1}$ such that $K \cap\left(V \times K_{1}\right)=V \times K^{*}$. By Corollary 4.6, $V \times K^{*} \in \operatorname{CIP}(G)$. Hence $K \in \operatorname{CIP}(K)$ and thus $H \in \operatorname{CIP}(G)$.

Corollary 4.15. Let $G \in[\mathrm{SIN}]$ be almost connected. Let $H$ be a closed abelian subgroup of $G$. Then $H \in \operatorname{CIP}(G)$.

Proposition 4.16. Let $G \in[\mathrm{SIN}]$ be almost connected. Let $V$ be a closed vector subgroup of $G$. Then $V \in \operatorname{CIP}(G)$.

Proof. As $G$ is almost connected, $G$ has a compact normal subgroup $K$ such that $G / K$ is an almost connected [SIN]-group [21, p. 698]. Since $V \cap K=$ $\{e\}, \varphi: G \rightarrow G / K$ is an isomorphism of $V$ onto $\varphi(V)$. However, $\varphi(V) \in$ CIP $(G / K)$ by Corollary 4.15 and the extending map can be lifted from $A(G / K)$ to $A(G)$.

Proposition 4.17. Let $G$ be an $[\mathrm{FC}]^{-}$group. Let $H$ be a closed subgroup of $G$ with no nontrivial compact subgroups. Then $H \in \operatorname{CIP}(G)$.

Proof. Let $G$ be an $\left[\mathrm{FC}^{-}\right.$group. Then $G$ has a compact normal subgroup $K$ such that $G / K$ is the direct product of a vector group and a discrete group. It follows that if $H \cap K=\{e\}$, then $H \in \operatorname{CIP}(G)$. 


\section{REFERENCES}

1. D. E. Alspace, $A$ characterization of the complemented translation invariant subspaces of $L_{1}\left(\mathbb{R}^{2}\right)$, J. London Math. Soc. 31 (1985), 115-124.

2. D. E. Alspach and A. Matheson, Projections onto translation-invariant subspaces of $L_{1}(\mathbb{R})$, Trans. Amer. Math. Soc. 77 (1984), 815-823.

3. D. E. Alspach, A. Matheson, and J. Rosenblatt, Projections onto translation-invariant subspaces of $L_{1}(G)$, J. Funct. Anal. 59 (1984), 254-292.

4. __ Separating sets by Fourier-Stieltjes transforms, preprint.

5. G. Arsac, Sur l'espace de Banach engendré par les coefficients d'une représentation unitaire, Thèse, Université Claude-Bernard, Lyon I, 1973.

6. L. Bagget and $\mathrm{K}$. Taylor, Groups with completely reducible regular representation, Proc. Amer. Math. Soc. 72 (1978), 593-600.

7. C. H. Chu, A note on scattered $C^{*}$-algebras and the Radon-Nikodym property, J. London Math. Soc. 24 (1981), 533-536.

8. J. Diestel and J. J. Uhl, Vector measures, Math. Surveys Monographs, no. 15, Amer. Math. Soc., Providence, RI, 1977.

9. P. Eymard, L'algèbre de Fourier d'un groupe localement compact, Bull. Soc. Math. France 92 (1964), 181-236.

10. B. Forrest, Amenability and bounded approximate identities in ideals of $A(G)$, Illinois J. Math. 34 (1990), 1-25.

11. E. Granirer and M. Leinert, On some topologies which coincide on the unit sphere of the Fourier-Stieltjes algebra $B(G)$ and of the measure algebra $M(G)$, Rocky Mountain J. Math. 11 (1981), 459-472.

12. C. Herz, Problems of extrapolation and spectral synthesis, Conference on Harmonic Analysis, Lecture Notes in Math., vol. 266, Springer-Verlag, New York, 1972.

13. __ Harmonic synthesis for subgroups, Ann. Inst. Fourier (Grenoble) 23 (1973), 91-123.

14. E. Hewitt and K. A. Ross, Abstract harmonic analysis, vol. I, Springer-Verlag, New York, 1963.

15. __, Abstract harmonic analysis, vol. II, Springer-Verlag, New York, 1963.

16. I. Khahil, Sur l'analyse harmonique du group affine de la droite, Studia Math. 51 (1974), 139-167.

17. V. Losert, On tensor products of Fourier algebra, Arch. Math. 43 (1984), 370-372.

18. G. W. Mackey, Induced representations of locally compact groups, Ann. of Math. 55 (1952), 101-140.

19. G. Mauceri, Square integrable representations and the Fourier algebra of a unimodular group Pacific J. Math. 73 (1977), 143-154.

20. D. J. Newman, The nonexistence of projections from $L^{1}$ to $H^{1}$, Proc. Amer. Math. Soc. 12 (1961), 98-99.

21. T. W. Palmer, Classes of nonabelian, noncompact, locally compact groups, Rocky Mountain J. Math. 8 (1978), 683-739.

22. H. P. Rosenthal, Projections onto translation invariant subspaces of $L^{p}(G)$, Mem. Amer. Math. Soc., No. 63, 1966.

23. W. Rudin, Projections on invariant subspaces, Proc. Amer. Math. Soc. 13 (1962), 429-432.

24. M. Takesaki and N. Tatsuuma, Duality and subgroups. II, J. Funct. Anal. 11 (1972), 184190.

25. K. Taylor, Geometry of the Fourier algebra and locally compact groups with atomic unitary representations, Math. Ann. 262 (1983), 183-190.

Department of Pure Mathematics, University of Waterloo, Waterloo, Ontario, Canada N2L 3G1 